\title{
Primary diffuse leptomeningeal gliomatosis: unusual MRI with non-enhancing nodular lesions on the cerebellar surface and spinal leptomeningeal enhancement
}

\author{
S Kastenbauer, A Danek, W Klein, T A Yousry, K Bise, G Reifenberger, H W Pfister
} Germany S Kastenbauer A Danek $\mathrm{H}$ W Pfister

Department of Neuroradiology T A Yousry

Institute of Neuropathology K Bise

Department of Neurology, Bezirkskrankenhaus Gabersee, Reitmehring, Germany W Klein

Department of Neuropathology, University of Bonn, Germany

G Reifenberger

Correspondence to: Dr Hans Walter Pfister Pfister@

nefo.med.uni-muenchen.de

Received 23 December 1999 and in revised form 13 April 2000

Accepted 13 April 2000

\begin{abstract}
Department of Neurology, Ludwig-Maximilians University, Marchioninistrasse 15, 81377 Munich,

Abstract

A 28 year old man presented with a 1 month history of symptoms of intracranial hypertension. Examination showed bilateral papilloedema and meningeal signs. Magnetic resonance imaging showed nodular lesions on the cerebellar and pontine surface and thickening of the thoracic spinal leptomeninges. Throughout the course of the disease, contrast enhancement was detected in the spinal leptomeninges but not intracranially. Primary diffuse leptomeningeal gliomatosis (PDLG) was diagnosed by biopsy and later confirmed on necropsy. The present case is remarkable for the nodular superficial cerebellar lesions and the absence of intracranial contrast enhancement of the leptomeninges.

(F Neurol Neurosurg Psychiatry 2000;69:385-388)
\end{abstract}

Keywords: primary diffuse leptomeningeal gliomatosis; chronic meningitis; magnetic resonance imaging

Glioma arising primarily from the leptomeninges is extremely rare and often is diagnosed only on postmortem examination. It is thought to derive from heterotopic nests of neuroglial tissue within the leptomeninges, which are present in $1 \%$ of necropsies. ${ }^{1}$ Primary leptomeningeal glioma can be in the form of a solitary tumour or a diffuse tumour involving intracranial or spinal cord leptomeninges. ${ }^{2}{ }^{3}$ Leptomeningeal gadolinium enhancement was positive in all cases of primary leptomeningeal glioma in which MRI was applied (eight cases on record). ${ }^{4-11}$ Brain MRI with gadolinium contrast enhancement is therefore considered the imaging modality of choice for the early detection of this condition. ${ }^{9}$ We report a case of primary diffuse leptomeningeal gliomatosis established by necropsy with the unusual MRI findings of nodular superficial cerebellar lesions and the absence of intracranial contrast enhancement of the leptomeninges.

\section{Case report}

In December 1997, a previously healthy 28 year old man developed frontal headaches.
From January 1998, the headaches were accompanied by nausea and vomiting. $\mathrm{He}$ became somnolent and was admitted to the Bezirkskrankenhaus Gabersee. On admission he was apathetic, confused, and had a slight neck stiffness. Otherwise, his neurological examination was normal; in particular he had no signs of spinal cord dysfunction. The patient was afebrile, and the blood chemistry results were normal. Cranial MRI showed multiple nodular hyperintensities (T2 weighted images, fig 1) on the cerebellar and pontine surface without any contrast enhancement ( $T 1$ weighted images, fig 1). The last follow up MRI with application of contrast medium was performed at the beginning of April. Until then, no intracranial contrast enhancement could be detected. On spinal MRI, leptomeningeal thickening and gadolinium enhancement were discernible from C7 to Th5 (fig 2). No lesion of the parenchyma of brain or spinal cord was detected on MRI then or later during the course of the disease. Analysis of CSF disclosed a pleocytosis of 30 cells $/ \mathrm{mm}^{3}$ (mainly lymphocytes and monocytes, cytology showed no malignant cells), a raised protein value $(4.2 \mathrm{~g} / \mathrm{l})$, and a glucose concentration of $2.55 \mathrm{mmol} / \mathrm{l}$ (glucose CSF/ serum index 0.5); oligoclonal bands were negative. The findings suggested a CNS infection and a broad antiviral (aciclovir) and antibacterial therapy (ceftriaxone, fosfomycin, ampicillin), including antituberculous drugs (isoniazid, rifampin, pyrazinamide) was started. However, CSF microscopy and cultures and serological tests did not confirm an infection. Therefore, the antimicrobial therapy was discontinued after 2 weeks. At the beginning of February 1998, the patient was released home. One week later, the headache increased again and he was admitted to our Neurological Department. Examination was normal except for bilateral papillodema. Repeated spinal taps showed raised CSF protein concentrations (up to $10 \mathrm{~g} / \mathrm{l}$ ) and a pleocytosis (up to 20 cells $/ \mathrm{mm}^{3} ; 20 \%$ lymphocytes, 35\% monocytes, $45 \%$ macrophages). Immunotyping showed no immature or atypical cells. Opening pressures were raised (300 to $860 \mathrm{~mm}$ 
$\mathrm{H}_{2} \mathrm{O}$ ) and were normalised by removal of 20-30 $\mathrm{ml}$ CSF, which greatly relieved the patient's headache. "Symptomatic" pseudotumour cerebri due to chronic meningitis was diagnosed and treated with repeated spinal taps. At the beginning of April 1998, the patient had a first grand mal seizure. Although phenytoin was started, the patient remained obtunded and his level of consciousness deteriorated to coma within hours. Both pupils were dilated and cranial CT showed discrete sulcal effacement of both cerebral hemispheres. A ventricular catheter was placed to measure intracranial pressure (ICP), which exceeded $40 \mathrm{~mm} \mathrm{Hg}$. After CSF drainage, osmotic therapy (mannitol and tris buffer), and hyperventilation, the patient regained consciousness within a few hours. Neurological status normalised except for left III and right VI cranial nerve palsy. A stereotactic biopsy of the surface of the cerebellum showed a leptomeningeal anaplastic astrocytoma corresponding to World Health Organisation (WHO) grade III. Two days after the biopsy, the patient had another episode of cerebral herniation with coma and wide, unresponsive pupils that required a decompressive craniotomy of the posterior fossa. Despite therapy, progressive brain swelling developed and the patient died after a 5 month course of illness.

Macroscopic postmortem examination showed diffuse tumour infiltration and thickening of the leptomeninges encasing the cervicothoracic spinal cord (fig $3 \mathrm{~A}$ ), brain stem, and cerebellum. There was a superficial nodular infiltration of the cerebellum. No parenchymal tumour was detected in the cerebrum, cerebellum, or spinal cord. Macroscopic and microscopic examination showed no heterotopic glial tissue in the intracranial or spinal leptomeninges. Microscopy confirmed a cellular glioma growing diffusely within the leptomeninges and subarachnoid space. The nodules in the cerebellar leptomeninges and the spinal leptomeningeal tumour were comparable in composition. The tumour cells showed an astro-
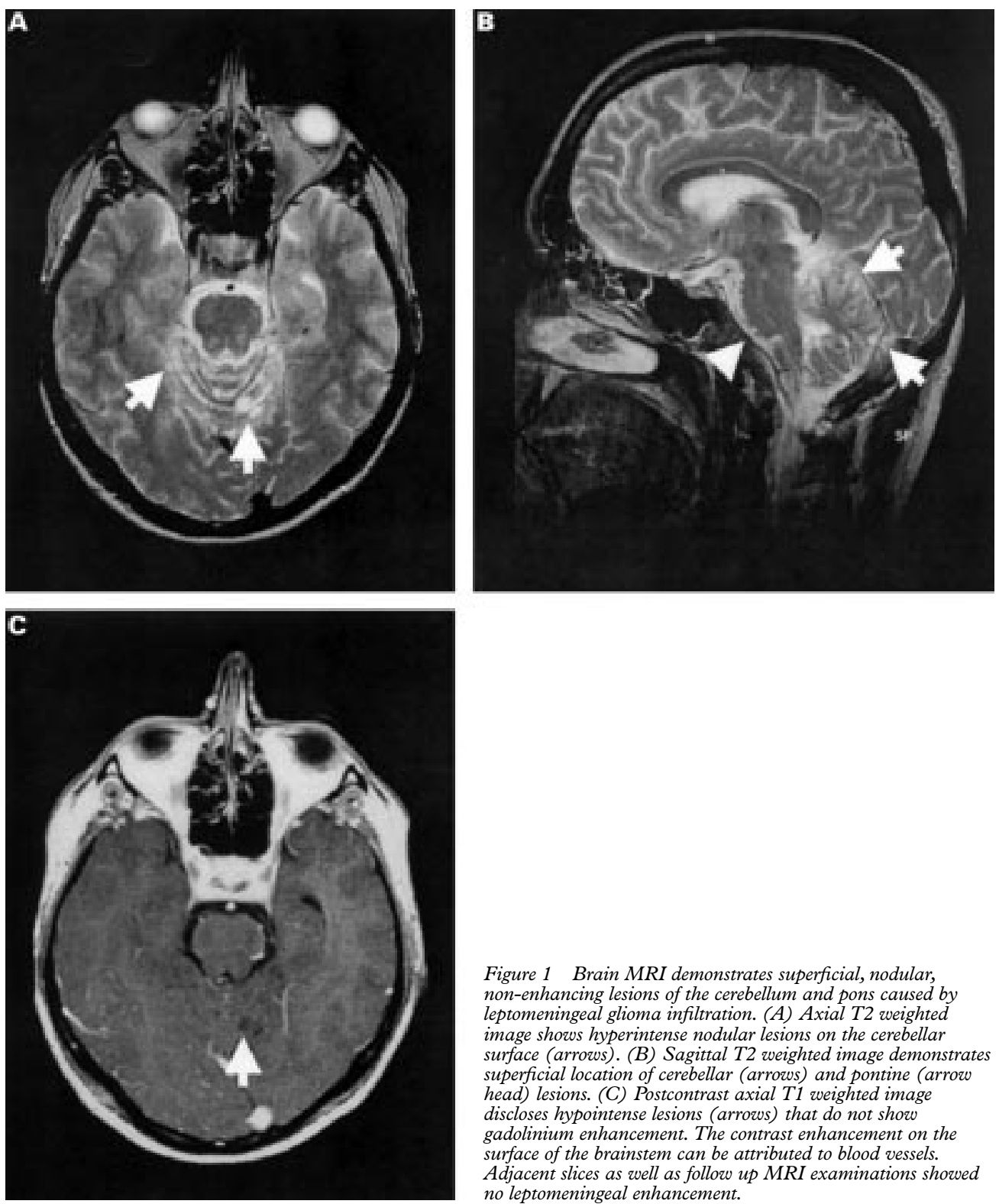

Figure 1 Brain MRI demonstrates superficial, nodular non-enhancing lesions of the cerebellum and pons caused by leptomeningeal glioma infiltration. (A) Axial T2 weighted image shows hyperintense nodular lesions on the cerebellar surface (arrows). (B) Sagittal T2 weighted image demonstrates superficial location of cerebellar (arrows) and pontine (arrow head) lesions. (C) Postcontrast axial T1 weighted image discloses hypointense lesions (arrows) that do not show gadolinium enhancement. The contrast enhancement on the surface of the brainstem can be attributed to blood vessels. Adjacent slices as well as follow up MRI examinations showed no leptomeningeal enhancement. 

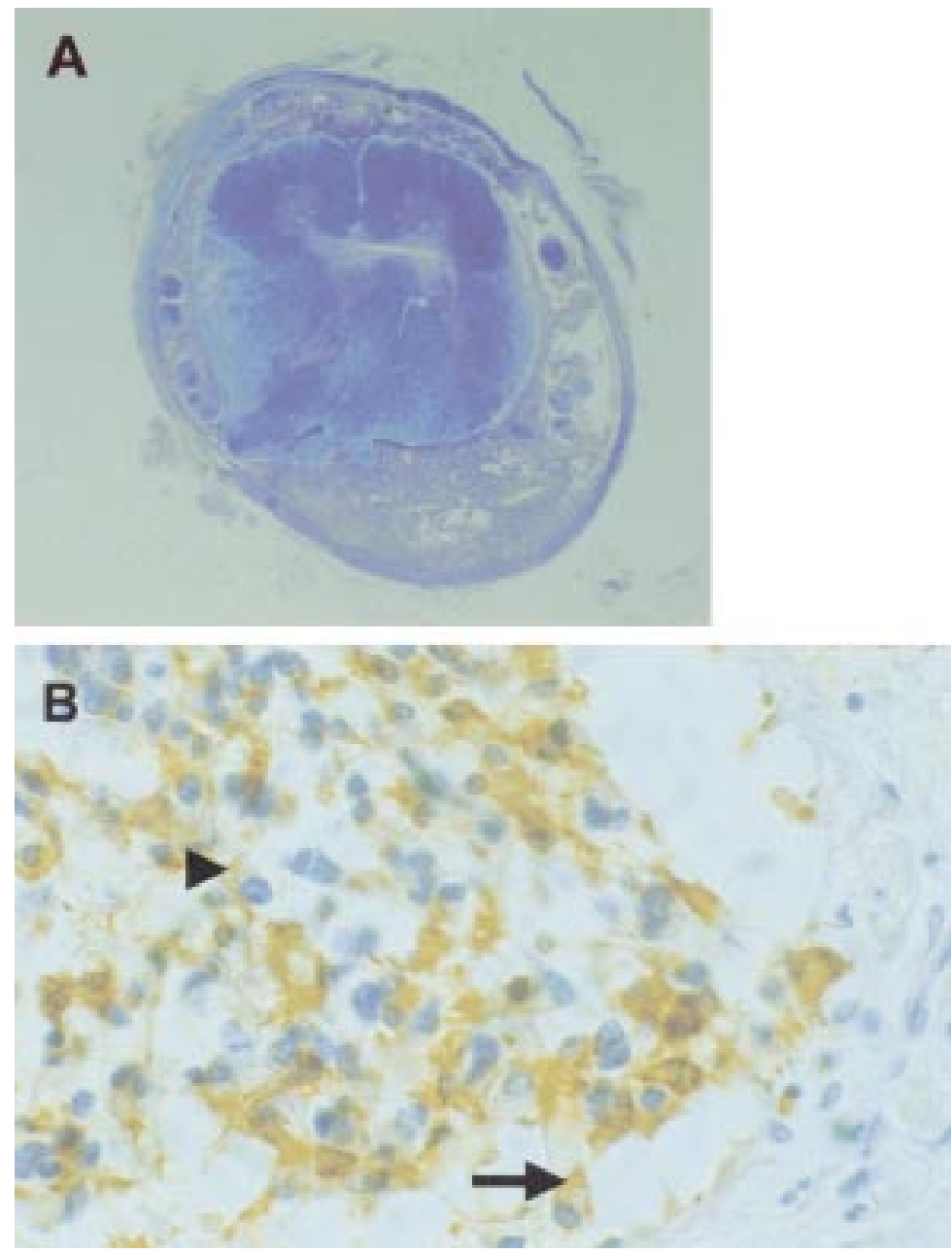

Figure 3 Cross section of the thoracic spinal cord. (A) Klüver-Barrera staining at low power magnification ( $3 \times$ ) demonstrates tumour masses infiltrating the leptomeninges and

subarachnoid space and encasing the spinal cord. Focal oedematous swelling of the subpial white matter can be seen. (B) Glial fibrillary acidic protein (GFAP) staining at high power magnification (400x). Tumour cells stain positive for GFAP to a high percentage (arrow). Nuclei are medium sized and of ovoid shape. Multipolar cytoplasmic processes are present (arrow head).

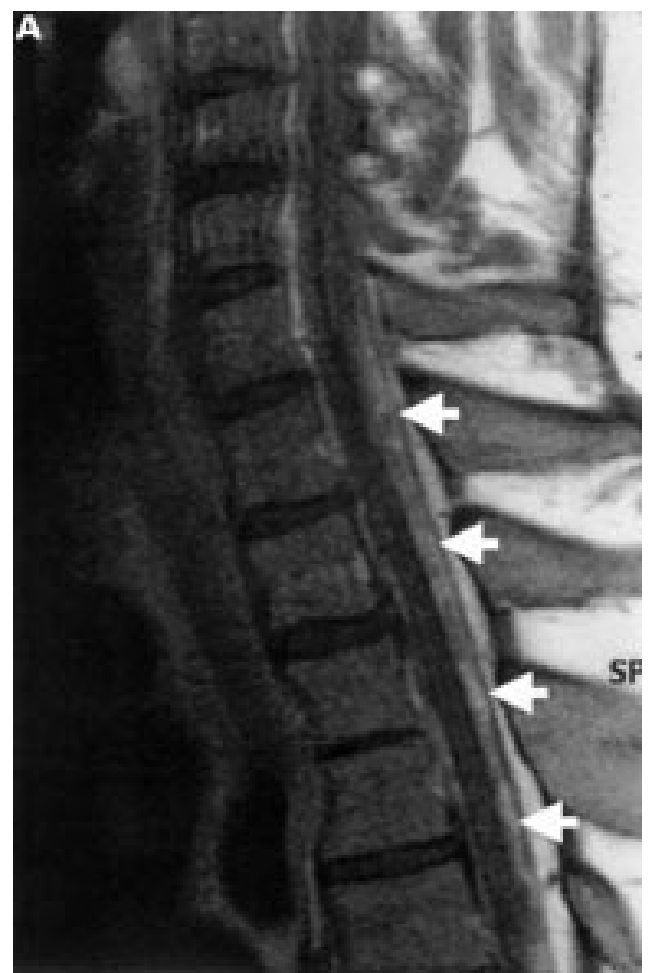

Figure 2 Cervical and thoracic spinal cord MRI demonstrating contrast enhancement of spinal leptomeningeal glioma. Postcontrast sagittal T1 weighted image shows gadolinium enhancement and thickening of the leptomeninges from $C 6 / 7$ to Th5 (arrows).

on the cerebellar surface caused us to suspect a parasitic infection such as echinococcosis or cysticercosis. However, the extensive search for an infectious aetiology was unsuccesful. Furthermore, repeated cytological CSF examination never showed malignant cells. Negative cytology seems to be a common finding in PDLG: only one of 24 cases on record had a positive cytology ${ }^{12}$ (retrieved by Medline and Pubmed search of English articles for the period 1966-99 and from publications cited therein). By contrast, eight of 12 patients with secondary meningeal tumour spread from intracranial malignant glioma were diagnosed antemortem by cytology. ${ }^{13}$ It remains unclear whether this discrepancy is due to a different biological behaviour of primary leptomeningeal gliomas or because CSF cytological examination is repeated less often or performed less thoroughly in PDLG than in patients with intracranial malignant glioma who are suspected to have secondary meningeal tumour spread. The neuroradiological findings of our case are remarkable, as a review of the literature showed that leptomeningeal thickening and contrast enhancement could be detected in six of eight cases of PDLG with cranial $\mathrm{CT}^{12}$ 14-18 and in all eight cases with MRI (cranial and/or spinal). ${ }^{4-11}$ Gadolinium enhanced MRI, therefore, was positive in all cases when applied, whereas contrast CT was read as negative in two of eight patients examined: in one patient no leptomeningeal enhancement was detected, ${ }^{19}$ and in the second enhancement was seen only retrospectively. ${ }^{20}$ Enhancement was either focal ${ }^{915}$ or, more often, diffuse in the spinal cord or brain. ${ }^{4-810-1216-1821}$ In addition to 
diffuse enhancement, small enhancing nodules of the spinal leptomeninges were reported in two cases. ${ }^{49}$ Nodular lesions, as seen in our patient, have not been reported so far. Furthermore, although the spinal tumour showed gadolinium enhancement, the intracranial tumour did not despite uniform histopathology. Therefore, contrast enhancement is not an obligatory feature of PDLG.

We thank Dr M Wick (Department of Clinical Chemistry), Dr W Stummer (Department of Neurosurgery), and Dr G Bachmann (Department of Neurology, Bezirkskrankenhaus Gabersee) for their cooperation

1 Cooper IS, Kernohan JW. Heterotopic glial nests in the subarachnoid space: histopathologic characteristics, mode of origin and relation to menigeal gliomas. $\mathcal{F}$ Neuropathol Exp

2 Ho KL, Hoschner JA, Wolfe DE. Primary leptomeningeal gliomatosis: symptoms suggestive of meningitis. Arch Neurol 1981;38:662-6.

$3 \mathrm{Ng} \mathrm{HK}$, Poon WS. Primary leptomeningeal astrocytoma Case report. F Neurosurg 1998;88:586-9.

4 Leproux F, Melanson D, Mercier C, et al. Leptomeningeal gliomatosis: MR findings. 7 Comput Assist Tomogr 1993;17: 317-20.

5 Krief O, Monnier L, Cornu P, et al. MR of isolated leptomeningeal glioma. Am f Neuroradiol 1994;15:1782-4.

6 Pingi A, Trasimeni G, Di Biasi C, et al. Diffuse leptomeninPeal gliomatosis with osteoblastic metastases and no geal gliomatosis with osteoblastic metastases and no 1018-20.

7 Giordana MT, Bradac GB, Pagni CA, et al. Primary diffuse leptomeningeal gliomatosis with anaplastic features. Acto Neurochir (Wien) 1995;132:154-9.
8 Kobayashi M, Hara K, Nakatsukasa M, et al. Primary spinal leptomeningeal gliomatosis presenting visual disturbance leptomeningeal gliomatosis presenting visual disturbance
as the initial symptom: case report. Acta Neurochir Wien as the initial sympt

9 Park JS, van den Noort S, Kim RC, et al. Primary diffuse leptomeningeal gliomatosis with signs of increased intracranial pressure and progressive meningeal enhancement on MRI. f Neuroimaging 1996;6:250-4.

10 Verslegers I, Demaerel P, Van Calenbergh F, et al. Primary multifocal leptomeningeal gliomatosis. Pediatr Radiol 1998; 28:580-2.

11 Paulino AC, Thomas C, Slomiany DJ, et al. Diffuse malignant leptomeningeal gliomatosis in a child. Am f Clin Oncol 1999;22:243-6.

12 Kitahara M, Katakura R, Wada T, et al. Diffuse form of primary leptomeningeal gliomatosis. Case report. $\mathcal{F}$ Neurosurg. 1985;63:283-7.

13 Yung WA, Horten BC, Shapiro WR. Meningeal gliomatosis: a review of 12 cases. Ann Neurol 1980;8:605-8.

14 Kalyan RU, Cancilla PA, Case MJ. Solitary, primary malignant astrocytoma of the spinal leptomeninges. $\mathrm{f} \mathrm{Neu}$ ropathol Exp Neurol 1983;42:517-21.

15 Wada C, Kurata A, Hirose R, et al. Primary leptomeningeal ependymoblastoma. Case report. I Neurosurg 1986;64: 968-73.

16 Rogers LR, Estes ML, Rosenbloom SA, et al. Primary leptomeningeal oligodendroglioma: case report. Neurosurgery 1995;36:166-8.

17 Beauchesne P, Pialat J, Duthel R, et al. Aggressive treatment with complete remission in primary diffuse leptomeningeal with complete remission in primary diffuse
gliomatosis. $\mathcal{F}$ Neurooncol 1998;37:161-7.

$18 \mathrm{Ng} \mathrm{HK}$, Poon WS. Diffuse leptomeningeal gliomatosis with oligodendroglioma. Pathology 1999;31:59-63.

19 Heye N, Iglesias JR, Tonsen K, et al. Primary leptomeningeal gliomatosis with predominant involvement of the spinal cord. Acta Neurochir Wien 1990;102:145-8.

20 Dietrich PY, Aapro MS, Rieder A, et al. Primary diffuse leptomeningeal gliomatosis (PDLG): a neoplastic cause of chronic meningitis. F Neurooncol 1993;15:275-83.

21 Bailey P, Robitaille Y. Primary diffuse leptomeningeal gliomatosis. Can f Neurol Sci 1985;12:278-81. 